\title{
OPEN Enhancement of Campylobacter hepaticus culturing to facilitate downstream applications
}

\begin{abstract}
Canh Phung ${ }^{1}$, Timothy B. Wilson ${ }^{2}$, José A. Quinteros ${ }^{2}$, Peter C. Scott ${ }^{2}$, Robert J. Moore ${ }^{1 \bowtie} \&$ Thi Thu Hao Van ${ }^{1}$

Campylobacter hepaticus causes Spotty Liver Disease (SLD) in chickens. C. hepaticus is fastidious and slow-growing, presenting difficulties when growing this bacterium for the preparation of bacterin vaccines and experimental disease challenge trials. This study applied genomic analysis and in vitro experiments to develop an enhanced $C$. hepaticus liquid culture method. In silico analysis of the anabolic pathways encoded by $C$. hepaticus revealed that the bacterium is unable to biosynthesise L-cysteine, L-lysine and L-arginine. It was found that L-cysteine added to Brucella broth, significantly enhanced the growth of $C$. hepaticus, but L-lysine or L-arginine addition did not enhance growth. Brucella broth supplemented with L-cysteine $(0.4 \mathrm{mM})$, L-glutamine $(4 \mathrm{mM})$, and sodium pyruvate $(10 \mathrm{mM})$ gave high-density growth of $C$. hepaticus and resulted in an almost tenfold increase in culture density compared to the growth in Brucella broth alone $(\log 10=9.3 \mathrm{vs} 8.4 \mathrm{CFU} / \mathrm{mL})$. The type of culture flask used also significantly affected $C$. hepaticus culture density. An SLD challenge trial demonstrated that $C$. hepaticus grown in the enhanced culture conditions retained full virulence. The enhanced liquid culture method developed in this study enables the efficient production of bacterial biomass and therefore facilitates further studies of SLD biology and vaccine development.
\end{abstract}

Campylobacter hepaticus has been identified as the causative agent of Spotty Liver Disease (SLD) in laying hens ${ }^{1}$. C. hepaticus is a fastidious bacterium that requires microaerobic conditions (a mix of 5-10\% oxygen, 5-10\% $\mathrm{CO}_{2}$, and $80-85 \% \mathrm{~N}_{2}$ ), a narrow temperature range (growth at 37 and $42{ }^{\circ} \mathrm{C}$, but not $25^{\circ} \mathrm{C}$ ), and rich nutrient media for growth ${ }^{1-4}$. The incubation time needed to form C. hepaticus colonies on agar plates varies from 3 to 7 days, depending on the strain cultivated ${ }^{1,2,5}$. The incubation time for other Campylobacter species, such as C. jejuni, C. coli, C. lari, and C. concisus ranges from 24 to $48 \mathrm{~h}$ on plates and $18-24 \mathrm{~h}$ in liquid media ${ }^{6-9}$. In previous studies, when a large $C$. hepaticus biomass was required, such as for SLD induction experiments, C. hepaticus was cultured on Brucella agar supplemented with 5\% defibrinated horse blood (HBA), incubated for 3 days and harvested. C. hepaticus had to be harvested from dozens of Petri dishes to produce sufficient biomass for a modestly sized animal trial ${ }^{10}$. This methodology is time-consuming, uses a lot of resources, and is prone to contamination. It also presents difficulties with scaling up to produce sufficient biomass to produce challenge material for large animal trials or for production of killed vaccines.

Although Brucella broth, the standard media for growing C. hepaticus cultures, is a rich medium, it may not supply all the nutrients required to support optimal growth of $C$. hepaticus. An in silico approach was used to identify nutrients that may need to be supplemented to improve culture productivity. The availability of C. hepaticus whole-genome sequences ${ }^{11}$ and tools such as Metagenomic Rapid Annotations using Subsystem Technology (MG-RAST) ${ }^{12}$ and Kyoto Encyclopedia of Genes and Genomes (KEGG) ${ }^{13}$ to annotate genomes and analyse metabolic pathways, allows the identification of growth-supporting compounds for C. hepaticus. KEGG analysis has been used to predict the nutritional requirements of $C$. jejuni NCTC $11168^{14}$. The study found that L-cysteine, L-leucine, L-methionine, and L-aspartic acid are essential amino acids that need to be exogenously supplied for the growth of $C$. jejuni. The addition of pyruvate or lactate and niacinamide as carbon sources have previously been shown to improve the growth of $C$. jejuni NCTC $11168^{15}$. Similarly, necessary substrates for the growth of Bukholderia glumae were defined using the Pathcomp tool in $\mathrm{KEGG}^{16}$.

The objective of this project was to identify and evaluate compounds that are required or that could enhance the growth of $C$. hepaticus in liquid culture, by analysing the metabolic pathways of this species. Also, culture conditions, including temperature, $\mathrm{pH}$, mixing, and culture vessel types were assessed to characterise and improve the growth of $C$ hepaticus in liquid culture. A reliable liquid culture method that resulted in high culture biomass

${ }^{1}$ School of Science, RMIT University, Bundoora West Campus, Bundoora, VIC, Australia. ${ }^{2}$ Scolexia Pty. Ltd., Moonee Ponds, VIC, Australia. ${ }^{\square}$ email: rob.moore@rmit.edu.au 


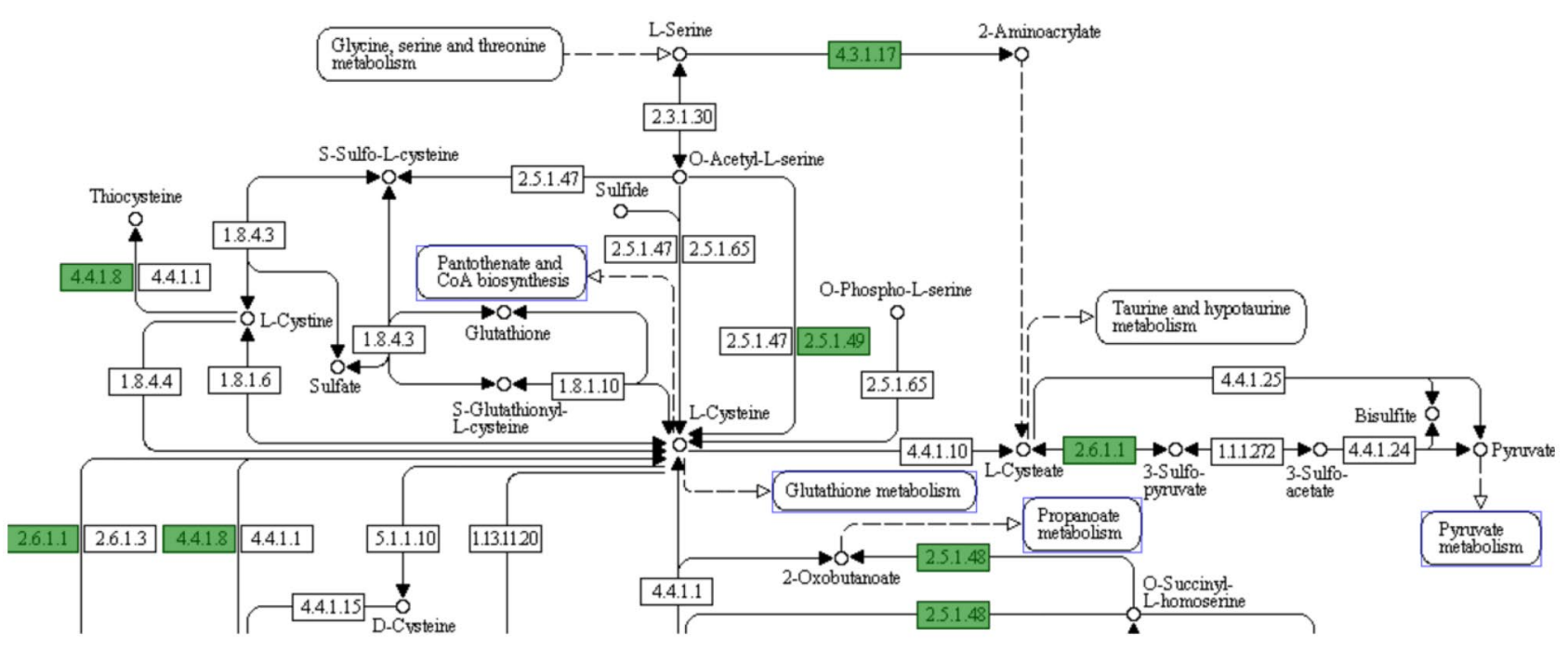

Figure 1. KEGG pathway of L-cysteine of C. hepaticus. Green boxes indicate the genes identified in $C$. hepaticus while white boxes show absent genes. C. hepaticus lacks genes including cysE (EC 2.3.1.30, serine acetyltransferase), cysK (EC 2.5.1.47, cysteine synthase A), cysM (EC 2.5.1.65, O-phosphoserine sulfhydrylase) and cys3 (EC 4.4.1.1, cystathionine $\gamma$-lyase).

would aid in the design of reproducible assays to investigate stress resistance, virulence mechanisms, vaccine development, and survival in the environment of $C$. hepaticus. This study applied genomic analysis and in vitro experiments to develop an enhanced $C$. hepaticus liquid culture method.

\begin{abstract}
Results
In silico pathway analysis of $C$. hepaticus. KEGG pathway analysis of three C. hepaticus strains showed that this bacterium harbours a complete tricarboxylic acid (TCA) cycle. Therefore, C. hepaticus can use all substrates in the citric pathways including pyruvate, succinate, oxaloacetate, fumarate, 2-oxoglutarate, malate and citrate as energy sources. The genome of $C$. hepaticus also contains genes encoding the enzymes for complete metabolic pathways for many amino acids, such as L-methionine, L-histidine, L-alanine, L-glycine, L-valine, $\mathrm{L}$-leucine and L-threonine. In contrast, $C$. hepaticus lacks a complete pathway for the biosynthesis of L-arginine from L-glutamate. The $\arg E$ gene, encoding acetylcitrulline deacetylase is absent. L-lysine cannot be biosynthesised from L-aspartate as the $\operatorname{dap} A$ and $d a p B$ genes are not present. Similarly, it is predicted that L-cysteine cannot be biosynthesised, as the $c y s E$, $c y s M$ and $c y s K$ genes required to synthesise L-cysteine from L-serine and L-methionine, are absent (Fig. 1). Consequently, C. hepaticus was predicted to be unable to biosynthesise L-cysteine, L-lysine and L-arginine. Adding these amino acids to the culture media may improve the growth of $C$. hepaticus. This prediction was tested in the following in vitro experiments. Sodium pyruvate was also added into Brucella broth to check the growth of $C$. hepaticus, because it has been used as a carbon source ${ }^{14}$ and scavenges hydrogen peroxide ${ }^{17}$, an oxidative stress factor that is generated via the use of oxygen of bacteria ${ }^{18}$.
\end{abstract}

The effect of culture vessel type, $\mathrm{pH}$, and inoculum level on the yield of $\boldsymbol{C}$. hepaticus. Before testing the effect of amino acids and sodium pyruvate on the growth of $C$. hepaticus, three factors, culture vessel types, $\mathrm{pH}$, and inoculum level were examined. Figure $2 \mathrm{~A}$ shows that $C$. hepaticus grew to a higher final density when growth in 24-well cell culture plates (CCP24) and $75 \mathrm{~cm}^{2}$ cell culture flasks (TCF75) $(\log 10 \mathrm{CFU} /$ $\mathrm{mL}=8.54-8.62(\mathrm{P} \leq 0.05)$ compared to that seen in $50 \mathrm{~mL}$ tubes (CT50) and $250 \mathrm{~mL}$ Erlenmeyer flasks (EF) $(\log \mathrm{CFU} / \mathrm{mL}=7.75-8.0)$. The CCP24 culture plates $(1 \mathrm{~mL}$ of culture added/well) were therefore used to test the effect of amino acid and sodium pyruvate supplementation on the growth of C. hepaticus, and TCF75 flasks (25 mL of media/flask) were used to scale-up the production of biomass of C. hepaticus.

C. hepaticus was monitored for growth in Brucella broth adjusted to a $\mathrm{pH}$ ranging from 6.0 to 8.0 to evaluate the $\mathrm{pH}$ tolerance. There was no statistically significant difference in the growth of $C$. hepaticus, indicating little or no effect of $\mathrm{pH}$ within this range (Fig. $2 \mathrm{~B}$ ). Therefore, Brucella broth without $\mathrm{pH}$ adjustment was used for the further culturing of $C$. hepaticus, as it has a neutral $\mathrm{pH}$ value $(7.0 \pm 0.2)$.

The growth kinetics of $C$. hepaticus in Brucella broth was investigated using initial inocula ranging from $10^{1}$ to $10^{7} \mathrm{CFU} / \mathrm{mL}$ (Fig. 2C). Viable cells counts were carried out after 0, 24, 48, 60, 72 and $96 \mathrm{~h}$ of incubation. The two highest inoculum levels $\left(10^{6}\right.$ and $\left.10^{7} \mathrm{CFU} / \mathrm{ml}\right)$ produced maximum growth to $10^{8} \mathrm{CFU} / \mathrm{ml}$ after $24 \mathrm{~h}$ of incubation. With the lower inoculum levels $\left(10^{5}\right.$ and $\left.10^{4} \mathrm{CFU} / \mathrm{mL}\right)$, C. hepaticus achieved maximum growth after $48 \mathrm{~h}$ of incubation and for low inoculum levels of $10^{1}$ to $10^{3} \mathrm{CFU} / \mathrm{ml}$, the maximum growth of C. hepaticus was reached after $60 \mathrm{~h}$.

The effect of amino acids and sodium pyruvate on the growth of $C$. hepaticus. C. hepaticus cultured in Brucella broth supplemented with $0.4 \mathrm{mM} \mathrm{L}$-cysteine, $4 \mathrm{mM} \mathrm{L}$ - glutamine, $0.8 \mathrm{mM}$ L-valine, $0.4 \mathrm{mM}$ $\mathrm{L}$-serine and $10 \mathrm{mM}$ sodium pyruvate exhibited a significantly greater growth compared to a culture grown in 
A
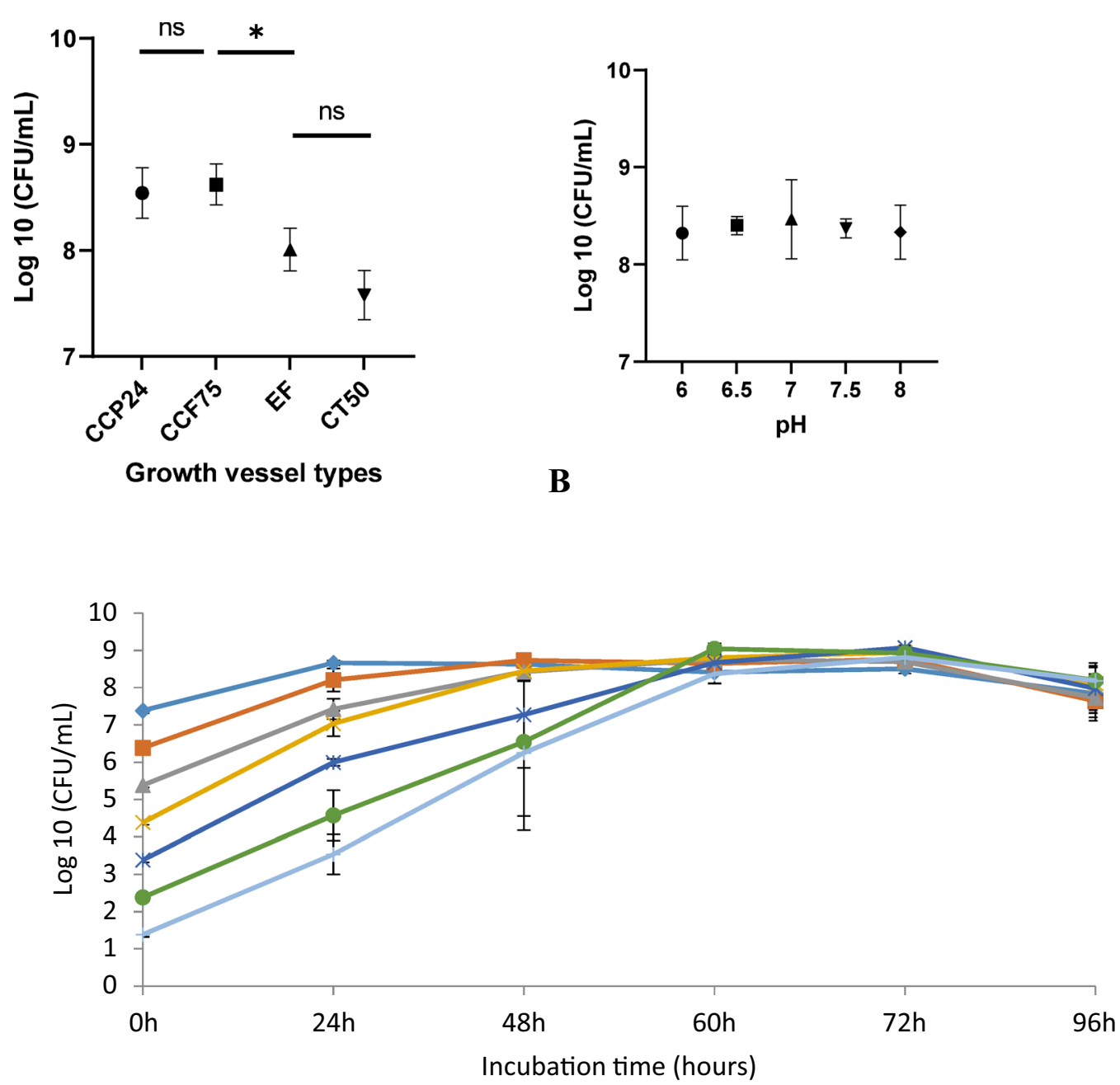

B

C

Figure 2. (A) Effect of growth vessel type on the growth of $C$. hepaticus $\mathrm{HV} 10^{\mathrm{T}}$. CCP24 24 well cell culture flask, TCF75 tissue culture flask $75 \mathrm{~cm}^{2}$, EF Erlenmeyer flask, CT50 corning $50 \mathrm{~mL}$ tube. (B) Growth of $C$. hepaticus in Brucella broth $\mathrm{pH}$ adjusted 6-8 after $48 \mathrm{~h}$ of incubation. (C) Kinetic growth of $C$. hepaticus in Brucella broth. The initial inoculum was from $10^{1}$ to $10^{7} \mathrm{CFU} / \mathrm{mL}$. Viable cells were counted after $0,24,48,60$, 72 and 96 h of incubation. P values were calculated using unpaired t-tests. Significant difference: ${ }^{\star} \mathrm{p} \leq 0.05, n s$ no significance. Data points represent results of 4 biological replicates.

Brucella broth only $(\log 10=8.66-9.26$ vs $8.31 \mathrm{CFU} / \mathrm{mL})$ (Fig. 3). L-cysteine $(0.4 \mathrm{mM})$ showed highest growth enhancement to $\log 10=9.26 \mathrm{CFU} / \mathrm{mL}$, significantly higher than the density obtained with the other supplements $(\mathrm{p} \leq 0.005)$. No significant difference was observed ( $p>0.05$, Fig. 3 ) when C. hepaticus was grown in Brucella broth supplemented with other amino acids including L-lysine, L-methionine, L-histidine, L-glycine, L- arginine, L-leucine, and L-threonine compared to a culture grown in Brucella broth only.

The growth of $C$. hepaticus was then investigated in Brucella broth supplemented with different combinations of each compound that could enhance the growth of C. hepaticus, as demonstrated above, including L-cysteine, L-glutamine, L-valine, L-serine, and sodium pyruvate. In general, all combinations of supplements in Brucella broth significantly enhanced the growth of $C$. hepaticus compared to Brucella broth alone. The highest growth of C. hepaticus observed was in Brucella broth supplemented with a mixture of $\mathrm{L}$-cysteine $(0.4 \mathrm{mM})$, L-glutamine $(4 \mathrm{mM})$ and sodium pyruvate $(10 \mathrm{mM})(\log 10=9.34)$, followed by a combination of $\mathrm{L}$-cysteine $(0.4 \mathrm{mM})$ and sodium pyruvate $(10 \mathrm{mM})(\log 10=9.17)$, and only L-cysteine $(0.4 \mathrm{mM})(\log 10=9.11)$ (Table 1$)$. Brucella broth supplemented with L-cysteine $(0.4 \mathrm{mM})$, and L-glutamine $(4 \mathrm{mM})$ and sodium pyruvate $(10 \mathrm{mM})$ was then used to grow $C$. hepaticus in TCF75 flasks in the following experiments.

Effect of temperature and incubating conditions on growth of $\boldsymbol{C}$. hepaticus. Because the culture vessels that provided the largest surface to volume ratio produced the highest density cultures it was proposed that gas exchange between the liquid medium and the microaerophilic atmosphere might be important. Therefore, the effects of agitation of the cultures by shaking were investigated. In the modified Brucella broth developed in this study, after $48 \mathrm{~h}$ of incubation C. hepaticus grew significantly better $(\mathrm{p} \leq 0.05)$ in static conditions, with $\log 10=9.25 \mathrm{CFU} / \mathrm{mL}$ compared to $\log 10=8.76 \mathrm{CFU} / \mathrm{mL}$ when shaken. It was also found that $C$. 


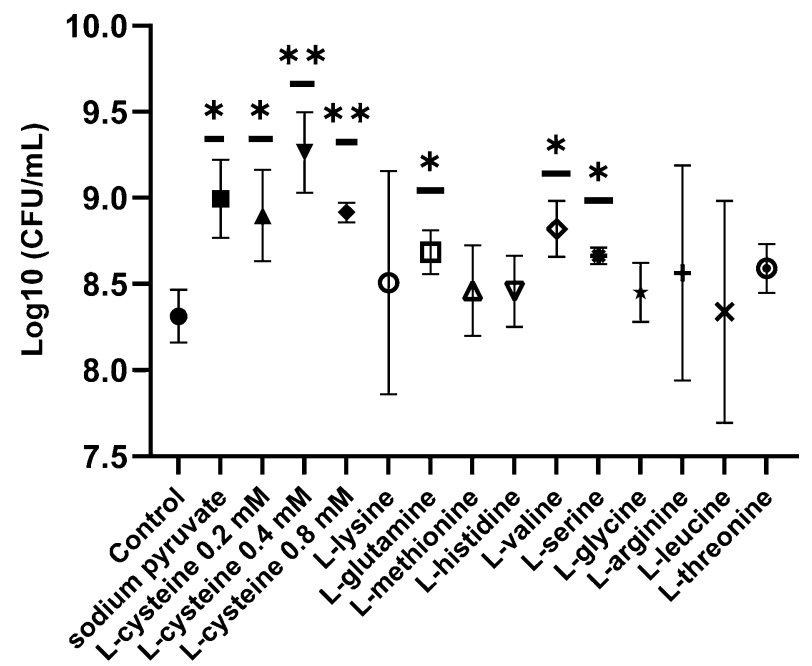

\section{Supplements}

Figure 3. Effect of sodium pyruvate and amino acids on the growth of C. hepaticus $\mathrm{HV} 10^{\mathrm{T}}$ in Brucella broth (control). Cultures were inoculated with $10^{6} \mathrm{CFU} / \mathrm{ml}$ and incubated for $48 \mathrm{~h}$ at $37^{\circ} \mathrm{C}$. P-values were calculated using unpaired t-tests. Significant difference ${ }^{\star}: \mathrm{p}<0.05{ }^{* *}: \mathrm{p}<0.005$.

\begin{tabular}{|l|l|l|l|l|}
\hline Medium & Log10 (CFU/mL) & SEM & P value & Significant difference \\
\hline Brucella broth (reference) & 8.42 & 0.10 & & \\
\hline Brucella broth + L-cysteine & 9.11 & 0.05 & $0.0008^{* * *}$ & Yes \\
\hline Brucella broth + L-cysteine + sodium pyruvate & 9.17 & 0.07 & $0.0009^{* * *}$ & Yes \\
\hline Brucella broth + L-cysteine + L-glutamine & 9.04 & 0.10 & $0.0049^{* *}$ & Yes \\
\hline Brucella broth + L-cysteine + L-glutamine + sodium pyruvate & 9.34 & 0.08 & $0.0003^{* * *}$ & Yes \\
\hline Brucella broth + L-cysteine + Sodium pyruvate + L-valine & 9.03 & 0.03 & $0.0012^{* *}$ & Yes \\
\hline Brucella broth + L-cysteine + Sodium Pyruate + L-serine & 9.01 & 0.18 & $0.0278^{*}$ & Yes \\
\hline
\end{tabular}

Table 1. The effects of different supplements added to Brucella broth on the growth of $C$. hepaticus compared to Brucella broth only. Cultures were inoculated with $10^{6} \mathrm{CFU} / \mathrm{ml}$ and incubated for $48 \mathrm{~h}$ at $37^{\circ} \mathrm{C}$. One way ANOVA followed by Dunnett's multiple comparison test showed that the differences between all the additive groups and the reference group (unsupplemented Brucella broth) were all highly significantly different. The $\mathrm{P}$-values presented are more conservative values calculated using unpaired t-tests. SEM standard error of mean, CFU colony-forming unit; significant difference. ${ }^{*}: \mathrm{p} \leq 0.05 ;{ }^{* *}: \mathrm{p} \leq 0.01{ }^{* * *}: \mathrm{p} \leq 0.001$.

hepaticus exhibited significantly better growth at $37^{\circ} \mathrm{C}$ than $42^{\circ} \mathrm{C}(\mathrm{p} \leq 0.05), \log 10=9.28 \mathrm{CFU} / \mathrm{mL}$ compared to $\log 10=8.09 \mathrm{CFU} / \mathrm{mL}$.

Growth of different $\boldsymbol{C}$. hepaticus strains in modified Brucella broth. The medium development experiments described above were carried out on a single strain of $C$. hepaticus. To determine if the improved media composition could also enhance the growth of other $C$. hepaticus isolates, the growth of different isolates in the modified Brucella broth, at $37^{\circ} \mathrm{C}$, under microaerobic conditions, in static TCF75 flasks was examined. All tested strains of C. hepaticus (HV10 ${ }^{\mathrm{T}}$, 19L. VICOCT18, WESTERN3, NSWJUNE19, SAJULY18 and DALE3) reached densities of $\log 10=9.25-9.49 \mathrm{CFU} / \mathrm{mL}$ after $48 \mathrm{~h}$, significantly higher $(\mathrm{p} \leq 0.05)$ than non-supplemented Brucella broth $(\log 10=8.24-8.66 \mathrm{CFU} / \mathrm{mL})$ Fig. 4). No significant difference in growth was observed among all C. hepaticus isolates in the modified Brucella broth.

Experimental infection of laying hens with $C$. hepaticus culture grown in modified Brucella broth. One of the principal reasons to develop improved culturing conditions for C. hepaticus was to make the preparation of challenge inocula easier and more reproducible for experimental infection trials used to investigate the biology of infections and assess the efficacy of various SLD treatment protocols. Experience with preparing challenge inocula using the previously described plate harvesting method had suggested that the state of the inoculum was critical to the success of infection ${ }^{10}$. Therefore, it was important to establish that the cultures grown in the modified Brucella liquid medium were capable of eliciting disease. Hens entering peak lay were challenged with, either C. hepaticus $\mathrm{HV} 10^{\mathrm{T}}$ harvested from Brucella agar plates, or bacteria grown in the newly devised liquid media conditions and the induction of SLD lesions on the liver were scored. Birds in the 


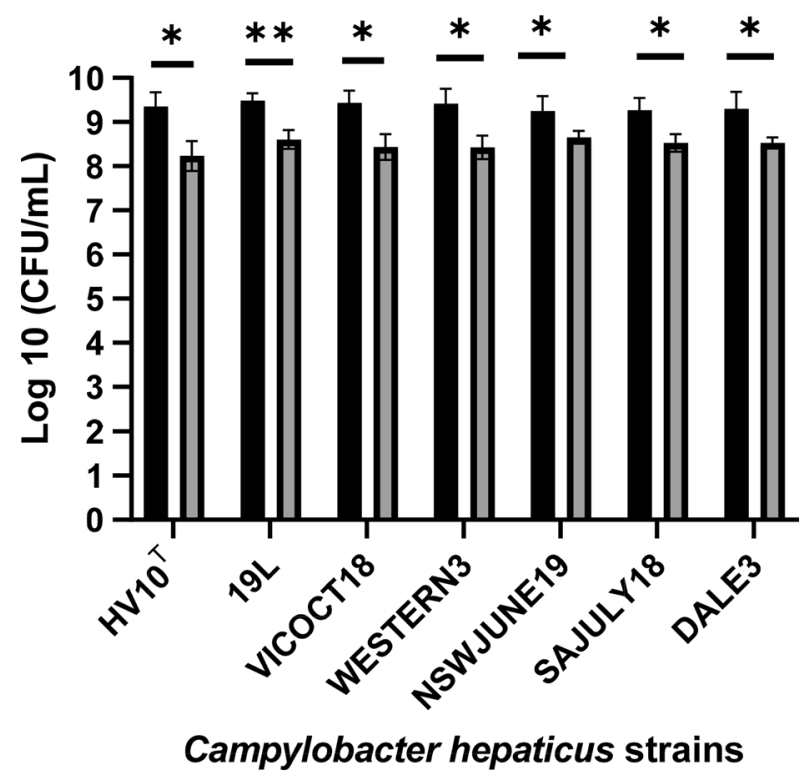

Figure 4. Growth of $C$. hepaticus isolates in modified Brucella broth (Brucella broth supplemented with $0.4 \mathrm{mM}$ L-cysteine, $4 \mathrm{mM}$ L-glutamine and $10 \mathrm{mM}$ sodium pyruvate) compared to non-supplemented Brucella broth after $48 \mathrm{~h}$ of incubation under microaerobic conditions. Solid black bars illustrate the growth of $C$. hepaticus strains in modified Brucella broth, grey bars in Brucella broth only. P values were calculated using unpaired t-tests. Significant difference ${ }^{\star}: \mathrm{p} \leq 0.05 .{ }^{\star *}: \mathrm{p} \leq 0.01$.

control group, inoculated with fresh modified Brucella broth, showed no lesion on livers, whereas 12 out of 12 hens inoculated with the agar plate derived bacteria had lesions on the liver and 11 out of 12 hens inoculated with bacteria grown in the modified Brucella liquid had liver lesions. Based on disease scores, there was no significant difference between the degree of disease elicited by bacteria grown under the two conditions (Fig. 5), demonstrating that $C$. hepaticus grown in the modified Brucella broth developed in this study had equal levels of virulence as those grown by the previously described plate culture method. The group sizes were sufficient to detect a one-point difference in mean scores with an alpha of 0.05 and $80 \%$ power.

\section{Discussion}

In silico pathway analysis of the $C$. hepaticus genome predicted that the bacterium lacks some of the genes encoding enzymes required for the biosynthesis of $\mathrm{L}$-cysteine, $\mathrm{L}$-lysine and $\mathrm{L}$-arginine. C. hepaticus lacks the genes cysE and $c y s K, c y s M$ to synthesise $\mathrm{L}$-cysteine from $\mathrm{L}$-serine and sulphur. $c y s E$ encodes serine acetyltransferase and $c y s K$ encodes cysteine synthase. These enzymes are required for the synthesis of $\mathrm{L}$-cysteine from $\mathrm{L}$-serine in bacteria and plants ${ }^{19}$. CysE synthesises L-cysteine from L-serine by catalysing an acyl transfer from acetyl-CoA. CysK catalyses O-acetyl-L-serine combining with hydrogen sulphide to yield $\mathrm{L}$-cysteine ${ }^{19}$. The addition of $\mathrm{L}$-cysteine to Brucella broth improved the growth of C. hepaticus significantly, to more than $10^{9} \mathrm{CFU} / \mathrm{mL}$ compared to Brucella broth only. This indicates that even though Brucella broth is a rich nutrient source the L-cysteine level is insufficient to support maximal growth of $C$. hepaticus. Growth support of $\mathrm{L}$-cysteine was also reported in other Campylobacter species. L-cysteine was defined as a vital source of sulphur for C. jejuni ${ }^{20}$. C. jejuni grown in minimal medium with L-cysteine added was better than their growth in the medium without cysteine ${ }^{14,21}$. $\mathrm{L}$-cysteine has been identified as a chemotactic attractants of $C$. jejuni ${ }^{22,23}$. In contrast, although C. hepaticus was also predicted to be unable to biosynthesise L-lysine and L-arginine, the addition of these amino acids to Brucella broth did not significantly increase the culture densities that could be achieved for $C$. hepaticus compared to densities supported by unmodified Brucella broth. Similarly, the addition of L-arginine and L-lysine to minimal media did not enhance the growth of $C$. jejuni because $C$. jejuni may have genes that support the biosynthesis of $\mathrm{L}$-arginine and $\mathrm{L}$-lysine ${ }^{14}$. Better growth of $C$. hepaticus in Brucella broth supplemented with L-glutamine, $\mathrm{L}$-valine or L-serine was observed although no missing genes that are responsible for the biosynthesis of these amino acids by $C$. hepaticus were detected. It was demonstrated that $\mathrm{L}$-glutamine and $\mathrm{L}$-serine are chemoattractants for C. jejuni ${ }^{24}$. In chickens, L-cysteine, $\mathrm{L}$-glutamine, and L-serine are abundant in chicken liver. The chicken gut also has sufficent quantities of necessary amino acids such as L-cysteine, L-glutamine, L-valine and L-serine for the growth of many bacteria ${ }^{25}$ and this likely explains why, despite its inability to synthesise a number of amino acids, C. hepaticus can colonise the intestinal tract of laying hens ${ }^{10}$. It was found that the reduction of amino acids such as L-cysteine, L-glutamine and L-serine in the chicken diet contributed to the reduction of Campylobacters in chickens ${ }^{26}$. The authors explained that these amino acid are involved in the formation of the mucin production of the intestinal mucus layer and essential for the survival and growth of Campylobacters ${ }^{27}$. Thus, an increase or decrease in the concentration of these amino acids results in changes in the number of Campylobacters in the chicken gut ${ }^{26}$. For other amino acids including L-methionine, L-histidine, L-glycine, 


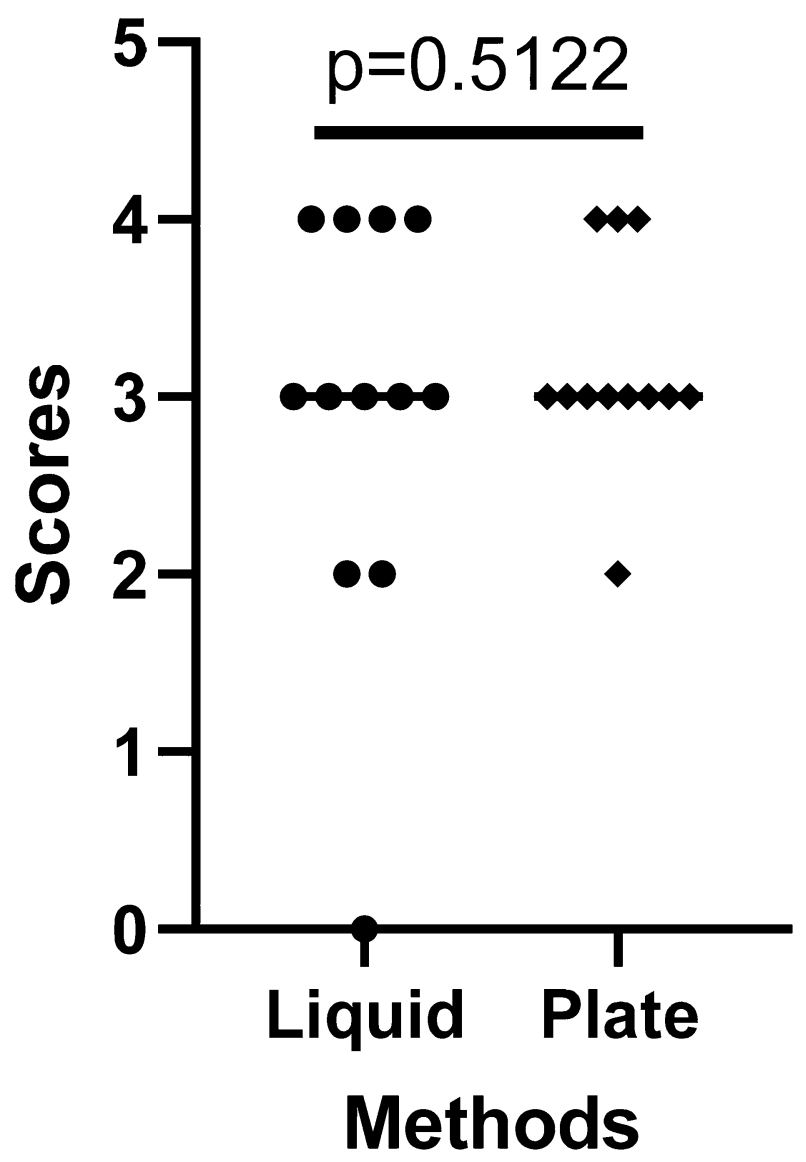

Figure 5. SLD spot scores of SLD-positive laying hens after challenge with C. hepaticus $\mathrm{HV} 10^{\mathrm{T}}$ grown from modified Brucella broth (liquid method) and HBA plate method based on scores of spots. This score is based on a logarithmic scale from 0 to 4 , where $0=$ no visible lesions, $1=1-9$ lesions, $2=10-99$ lesions, $3=100-999$ lesions and $4=$ more than 1000 lesions. $P$ values were calculated using unpaired t-tests.

L-leucine, and L-threonine, there was no significant difference in the growth of $C$. hepaticus. These results agree with the bioinformatic pathway analysis.

This study found that the $C$. hepaticus showed maximum growth in Brucella broth supplemented with a mixture of L-cysteine $(0.4 \mathrm{mM})$, L-glutamine $(4 \mathrm{mM})$ and sodium pyruvate $(10 \mathrm{mM})$ and reached $10^{9.34} \mathrm{CFU} /$ $\mathrm{mL}$. Sodium pyruvate provides an additional carbon source to support the growth of $C$. hepaticus in Brucella broth. C. hepaticus may heavily depend on the Kreb's cycle to generate energy. Many studies have demonstrated the important role of pyruvate in the growth of campylobacters. Sodium pyruvate is one of the compounds in the Campylobacter growth supplement product (FBP supplement ${ }^{28}$ and Campylobacter selective supplement ${ }^{29}$. Pyruvate was found to promote the growth of $C$. jejuni NCTC 11168 in MEM medium ${ }^{14}$. This compound plays a central role in C. jejuni metabolism and can be fermented to various products such as acetate, formate, lactate and succinate. It is linked to carbohydrate and amino acid catabolism to produce energy. The synthesis of L-leucine, $\mathrm{L}$-valine, L-alanine and L-isoleucine by campylobacters use pathways in which pyruvate can play a key role ${ }^{30}$. Pyruvate also acts as an electron acceptor and can decrease the concentration of hydrogen peroxide to reduce the damage caused by oxygen to bacteria ${ }^{31}$.

C. hepaticus grew to higher densities in CCP24 and TCF75 than CT50 and EF, suggesting that the surface-area-to-volume ratio $(\mathrm{S}: \mathrm{V})$ may be important, possibly for gas exchange, as mentioned in a study of improvement of culturing of $C$. jejuni ${ }^{32}$. These authors demonstrated that TCF75 has more S:V ratio than EF and CT50 and therefore TCF75 provided better atmospheric exchange than EF and CT50. Also, tissue culture flasks were recommended to grow and study standard growth curves of C. jejuni ${ }^{33}$. In a study of the growth of Helicobacter pylori, Gas-Permeable Lifecell tissue culture flasks gave improved growth in Brucella broth supplemented with fetal bovine serum ${ }^{34}$. These authors also mentioned the effect of surface area on the growth of $H$. pylori and suggested that a small surface area resulted in poorer growth of $H$. pylori. Tissue culture flasks were used early in the history of the culture of C. jejuni and C. pylori $i^{35,36}$ and have been employed in many studies of Campylobacter species. It may show that $C$. hepaticus requires a larger $\mathrm{S}: \mathrm{V}$ ratio to grow due to better gas exchange, although it is then unexpected that agitation had a negative effect. A low level of oxygen may be an obligatory requirement for C. hepaticus growth. Under anaerobic conditions and higher oxygen tensions (21\%), C. hepaticus failed to grow ${ }^{1}$. Similarly, C. jejuni does not grow under anaerobic conditions ${ }^{37,38}$ and oxygen is considered a requirement for 
DNA synthesis in C. jejuni ${ }^{37}$. Campylobacter spp. are sensitive to high oxygen tensions, but still need an optimal oxygen concentration ( $2 \%-10 \%)$ to grow ${ }^{38}$. C. hepaticus contains genes for oxidative phosphorylation ${ }^{11}$ that need oxygen as an acceptor to generate ATP.

C. hepaticus achieved maximum growth after 48-72 h, depending on the initial inoculum, showing slower growth in comparison to other Campylobacters. The maximum growth of $C$. jejuni was at around $30 \mathrm{~h}$ in both Nutrient Broth Number 2 and Mueller Hinton broth ${ }^{39}$. C. jejuni reached the highest densities after 24 h of growth in Brain Heart Infusion broth ${ }^{40}$. C. hepaticus can grow in a range of $\mathrm{pH}$ from 6.0 to 8.0. It has been reported that other Campylobacter species also grow well in this $\mathrm{pH}$ range $\mathrm{e}^{41}$. Chickens normally have a $\mathrm{pH}$ of around 6.3 in the liver ${ }^{42}, 6.4$ in the small and large intestine ${ }^{43}, 6.6-6.7$ for caecum ${ }^{43,44}$, and 6.0 for bile ${ }^{45}$. These are all tissues and environments in which it has been shown that $C$. hepaticus can survive and colonise. C. hepaticus showed better growth at $37^{\circ} \mathrm{C}$ than $42^{\circ} \mathrm{C}$ while this microorganism has only been isolated from chickens, which have a body temperature of $40-42^{\circ} \mathrm{C}^{46}$. The growth of $\mathrm{C}$. hepaticus was reported to be somewhat slow at $42^{\circ} \mathrm{C}$, taking 7 days to form colonies in sheep blood agar ${ }^{3}$. Temperature differently affects Campylobacter species regarding the growth, motility, and ability to invade the host cells. A study showed that $C$. jejuni grew at both $37^{\circ} \mathrm{C}$ and $42^{\circ} \mathrm{C}$ but showed differences in motility and invasion. C. coli grew and moved better at $42^{\circ} \mathrm{C}$. C. fetus, a bacterium that is frequently detected in poultry, showed greater growth and invasion at $37^{\circ} \mathrm{C}^{47}$.

Using the modified Brucella broth developed in this study, together with growth conditions including the use of a large surface area culture vessel, at $37^{\circ} \mathrm{C}$, in microaerophilic and static conditions, $\mathrm{C}$. hepaticus cultures could grow to $10^{9} \mathrm{CFU} / \mathrm{mL}$ and showed virulence in laying hens. This culturing method is time-saving and more cost-effective than the previously used plate harvesting method to obtain the large biomass required for SLD animal induction experiments and bacterin vaccine production. It also reduces the amount of subculturing needed, possibly minimising the effect of repeated subculture on the virulence of $C$. hepaticus. A study has shown that the repeated subculturing of the somewhat related organism, $H$. pylori, could result in a decrease of adhesion, motility, gastric inflammation and cytotoxicity, and repeated culturing is a recognised way that bacteria have been attenuated to produce live vaccines ${ }^{48}$. Thus, the method described in this study can facilitate further studies on C. hepaticus biology and SLD.

\section{Materials and methods}

In silico analysis of $C$. hepaticus metabolism. The metabolic pathways encoded within the genomes of C. hepaticus strains HV10 ${ }^{\mathrm{T}}$, 19L and VICOCT18, were analysed using (1) RAST (Rapid Annotation using Subsystem Technology) ${ }^{12}$ and the $\mathrm{SEED}^{49}$ (http://rast.nmpdr.org/rast.cgi) for annotating the genomes and metabolic pathways prediction of C. hepaticus; and (2) BLAST ${ }^{50}$ (Basic Local Alignment Search Tool) (https://blast.ncbi. nlm.nih.gov/Blast.cgi) for comparing nucleotides and protein sequences of $C$. hepaticus with available sequences in the gene bank. C. hepaticus $\mathrm{HV} 10^{\mathrm{T}}$ is the type strain for C. hepaticus, $19 \mathrm{~L}$ is a representative of a clade that is distinct form $\mathrm{HV} 10^{\mathrm{T}}$ and VICOCT18 is a more recent isolate.

Bacterial strains and culture conditions. The C. hepaticus strains HV10 ${ }^{\mathrm{T}}, 19 \mathrm{~L}^{11}$, VICOCT18, WESTERN3, NSWJUNE19, SAJULY18 and DALE3 ${ }^{51}$ were used in the study. These strains are representative isolates from independent SLD outbreaks from widely separated geographical locations in Australia. All C. hepaticus strains were stored at $-80{ }^{\circ} \mathrm{C}$ in $70 \%$ Brucella broth $\left(\mathrm{BD} \mathrm{BBL}^{\mathrm{su}}\right.$ ) and $30 \%$ glycerol. C. hepaticus was routinely cultured on Brucella agar plates (Brucella broth $\left(\mathrm{BD} \mathrm{BBL}^{\mathrm{ru}}\right)+1.5 \%$ agar $\left(\mathrm{BD} \mathrm{BBL} \mathrm{BL}^{\mathrm{m}}\right)$ ) supplemented with $5 \%$ defibrinated horse blood (Equicel) (HBA) and cultured at $37^{\circ} \mathrm{C}$ under microaerobic conditions (created using Campygen 3.5L gas generation packs (Oxoid)) in an anaerobic jar, for $96 \mathrm{~h}$ to recover C. hepaticus cells from $-80^{\circ} \mathrm{C}$ stock or for $72 \mathrm{~h}$ if they were subcultured from HBA plates.

Effect of type of culture vessel on the growth of $\boldsymbol{C}$. hepaticus. Costar 24 -well cell culture plates (CCP24), Corning $50 \mathrm{~mL}$ centrifuge tubes (CT50) with a vented cap $\left(0.2 \mu \mathrm{m}\right.$ pore size), Corning $75 \mathrm{~cm}^{2}$ cell culture flask (TCF75) with a vented cap $(0.2 \mu \mathrm{m}$ pore size) and Erlenmeyer flasks $(250 \mathrm{~mL})(\mathrm{EF})$ were used to compare the growth of $C$. hepaticus in Brucella broth $\left(\mathrm{BD} \mathrm{BBL}^{\mathrm{sm}}\right)$. The volume of culture media used in CCP24 was $1 \mathrm{~mL}, 25 \mathrm{~mL}$ (CT50 and TCF) and $40 \mathrm{~mL}$ (EF). All vessels were placed in BD GasPak ${ }^{\mathrm{rm}}$ EZ container, charged with CampyGen $3.5 \mathrm{~L}$ (Oxoid) to produce microaerobic conditions and then incubated at $37^{\circ} \mathrm{C}$ for $48 \mathrm{~h}$. The growth rate of $C$. hepaticus $\mathrm{HV} 10$ was determined by the plate count method on HBA plates.

Effect of $\mathrm{pH}$ and initial inoculum on $\mathrm{C}$. hepaticus growth. The $\mathrm{pH}$ of Brucella broth was adjusted from 6.0 to 8.0 in increments of 0.5 units using $1 \mathrm{M} \mathrm{NaOH}$ or $1 \mathrm{M} \mathrm{HCl}$. C. hepaticus was cultured in CCP24 plates to examine growth at different $\mathrm{pH}$ levels. Each well of CCP24 was inoculated with $10^{6} \mathrm{CFU} / \mathrm{mL}$ of $C$. hepaticus $\mathrm{HV} 10^{\mathrm{T}}$. Plates were incubated under microaerobic conditions at $37^{\circ} \mathrm{C}$. Growth was enumerated after $48 \mathrm{~h}$ of incubation by plating serial dilution on HBA. The experiment was performed in triplicate.

The effect of initial inoculum size on the growth kinetics and final culture yields was analysed using seven initial inoculum levels $\left(10^{1}\right.$ to $\left.10^{7} \mathrm{CFU} / \mathrm{ml}\right)$ of $C$. hepaticus $\mathrm{HV} 10^{\mathrm{T}}$ in a CCP24 plate. The plates were incubated at $37^{\circ} \mathrm{C}$ under microaerobic conditions. The growth of $C$. hepaticus $\mathrm{HV} 10^{\mathrm{T}}$ was examined after $24,48,60,72$, and $96 \mathrm{~h}$ of incubation using the plate count method on HBA.

Growth of C. hepaticus in static and shaking conditions. C. hepaticus $\mathrm{HV} 10^{\mathrm{T}}$ was suspended into Brucella broth to achieve acell density of $10^{5} \mathrm{CFU} / \mathrm{ml}$ and then $1 \mathrm{~mL}$ of bacterial suspension was incubated into each well of CCP24 at static condition. For shaking conditions, the anaerobic jars were shaken in a shaking incubator with a speed of $100 \mathrm{rpm}$. After $48 \mathrm{~h}$ viable C. hepaticus cells were enumerated on HBA plates. The shaking conditions were only used for this test; static growth conditions were used at all other times. 
Effects of sodium pyruvate and amino acids on the growth of $C$. hepaticus. Based on the results of in silico pathway analysis of C. hepaticus and results from Alazzam et al. ${ }^{14}$, the following supplements were added to Brucella broth to examine the growth of this bacterium: amino acids (L-cysteine, L-lysine, L-methionine, and L-leucine, L-glutamine, L-valine, L-histidine); carbon source (sodium pyruvate) (Sigma). Each compound was completely dissolved in Milli-Q water at a concentration recommended in MCLMAN, a new minimal medium for C. jejuni $i^{14}$. The chemical solution was passed through a $0.22 \mu \mathrm{m}$ syringe filter and stored at $-20^{\circ} \mathrm{C}$ if not used immediately. On the day of the experiment, each substrate was added into a bacterial culture and then $1 \mathrm{~mL}$ transferred to each well of CCP plates. The plates were incubated under microaerobic conditions at $37^{\circ} \mathrm{C}$ for $48 \mathrm{~h}$.

Effect of temperature on growth of $C$. hepaticus. Growth of $C$. hepaticus was tested at $37^{\circ} \mathrm{C}$ and $42{ }^{\circ} \mathrm{C}$ in Brucella broth. Briefly, C. hepaticus cells were harvested from an HBA plate and suspended into Brucella broth to obtain an $\mathrm{OD}$ of $0.01-0.03$. The bacterial suspension was supplemented with sodium pyruvate $(10 \mathrm{mM})$, L-cysteine $(0.4 \mathrm{mM})$, and L-glutamine $(4 \mathrm{mM})$ (based on the results from the experiments described above). Bacterial culture $(1 \mathrm{~mL})$ was placed in CCP plates and incubated under microaerobic conditions for $48 \mathrm{~h}$ at $37^{\circ} \mathrm{C}$ and $42^{\circ} \mathrm{C}$.

Animal trial. A C. hepaticus chicken challenge trial was carried out to compare the virulence between $C$. hepaticus $\mathrm{HV} 10^{\mathrm{T}}$ cultures grown from modified Brucella broth developed in this study (liquid method) and the HBA plates (plate method). The plate method was described by Van et al. ${ }^{10}$ in which C. hepaticus $\mathrm{HV} 10^{\mathrm{T}}$ stored at $-80^{\circ} \mathrm{C}$ in glycerol stocks was first streaked on to HBA plates, then further subcultured to HBA plates and cells were harvested and resuspended in Brucella broth to obtain $1 \times 10^{9} \mathrm{CFU} / \mathrm{mL}$. For the liquid method, C. hepaticus was grown in Brucella broth supplemented with L-cysteine $(0.4 \mathrm{mM})$, and L-glutamine $(4 \mathrm{mM})$ and sodium pyruvate $(10 \mathrm{mM})$ in TCF 75 at $37^{\circ} \mathrm{C}$ for $48 \mathrm{~h}$ in microaerophilic conditions and used directly for the challenge. The animal experimentation was approved by the Wildlife and Small Institutions Animal Ethics Committee of the Victorian Department of Economic Development, Jobs, Transport and Resources (approval number 14.16). A total of 36 Hy-Line brown laying hens were used in the experiment. Only healthy birds laying eggs regularly were included in the study. Chickens were housed in groups of 4 birds per pen, with 3 pens per group: a total of 12 birds in each group $(n=12)$. Birds were randomly allocated to groups and cages by stratified rank order based on weight. Unchallenged control birds were orally inoculated with $1 \mathrm{ml}$ of Brucella broth only whereas $1 \mathrm{ml}$ of Brucella broth containing $1 \times 10^{9} \mathrm{CFU}$ of $\mathrm{C}$. hepaticus $\mathrm{HV} 10^{\mathrm{T}}$ was orally administered to the birds for the plate and broth methods groups. Birds were sacrificed after 5 days and SLD lesions on the surface of the liver were counted to measure the severity of the induced disease. The experienced and trained scorers were blinded to the treatment groups. Scores were based on a logarithmic scale from 0 to 4 , where $0=$ no visible lesions, $1=1-9$ lesions, $2=10-99$ lesions, $3=100-999$ lesions and $4=$ more than 1000 lesions.

Statistical analysis. All experiments to study the growth of C. hepaticus HV10 in different conditions and supplements were repeated a minimum of three times with biological replicates. Campylobacter cell counts in all tests were converted to $\log _{10} \mathrm{CFU} / \mathrm{mL}$. Statistical comparison of all parameters was performed by t-test, one-way ANOVA using Graphpad Prism version 8 for Windows, GraphPad Software (San Diego California USA, www. graphpad.com). The significance level was set at $5 \%(\mathrm{p} \leq 0.05)$. Sample size calculation for the SLD animal trial was performed using the online calculator at https://clincalc.com/stats/samplesize.aspx.

Declarations. All the methods were carried out in accordance with relevant guidelines and regulations and the animal trial is reported in accordance with the ARRIVE Essential 10 guidelines.

Received: 5 July 2021; Accepted: 30 September 2021

Published online: 21 October 2021

\section{References}

1. Van, T.T.H., Elshagmani, E., Gor, M.C., Scott, P.C. \& Moore, R.J. Campylobacter hepaticus sp. nov., isolated from chickens with spotty liver disease. Int. J. Syst. Evol. Microbiol. 66, 4518-4524. https://doi.org/10.1099/ijsem.0.001383 (2016).

2. Crawshaw, T. R. et al. Isolation of a novel thermophilic Campylobacter from cases of spotty liver disease in laying hens and experimental reproduction of infection and microscopic pathology. Vet. Microbiol. 179, 315-321. https://doi.org/10.1016/j.vetmic.2015. 06.008 (2015).

3. Gregory, M., Klein, B., Sahin, O. \& Girgis, G. Isolation and characterization of Campylobacter hepaticus from layer chickens with spotty liver disease in the United States. Avian. Dis. 62, 78-85. https://doi.org/10.1637/11752-092017-Reg.1 (2018).

4. Crawshaw, T. R. et al. Isolation of Campylobacter hepaticus from free-range poultry with spotty liver disease in New Zealand. N. Z. Vet. J. 69, 58-64. https://doi.org/10.1080/00480169.2020.1801532 (2020).

5. Grimes, T. \& Reece, R. Spotty liver disease-An emerging disease in free-range egg layers in Australia. in Proceedings of the Sixtieth Western Poultry Disease Conference. 53-56 (2011).

6. Khan, I. U. H., Hill, S., Nowak, E. \& Edge, T. A. Effect of incubation temperature on the detection of thermophilic Campylobacter species from freshwater beaches, nearby wastewater effluents, and bird fecal droppings. Appl. Environ. Microbiol. 79, $7639-7645$. https://doi.org/10.1128/AEM.02324-13 (2013).

7. Kim, J. et al. An improved culture method for selective isolation of Campylobacter jejuni from wastewater. Front. Microbiol. 7, 1345. https://doi.org/10.3389/fmicb.2016.01345 (2016).

8. Ismail, Y., Lee, H., Riordan, S. M., Grimm, M. C. \& Zhang, L. The effects of oral and enteric Campylobacter concisus strains on expression of TLR4, MD-2, TLR2, TLR5 and COX-2 in HT-29 cells. PLoS ONE 8, e56888. https://doi.org/10.1371/journal.pone. 0056888 (2013).

9. Reilly, S. S. \& Gilliand, S. E. Improved culturing techniques for Campylobacter. J. Food Sci. 68, 2752-2757 (2003). 
10. Van, T. T. H. et al. Induction of spotty liver disease in layer hens by infection with Campylobacter hepaticus. Vet. Microbiol. 199, 85-90. https://doi.org/10.1016/j.vetmic.2016.12.033 (2017).

11. Van, T. T. H. et al. Survival mechanisms of Campylobacter hepaticus identified by genomic analysis and comparative transcriptomic analysis of in vivo and in vitro derived bacteria. Front. Microbiol. 10, 107-107. https://doi.org/10.3389/fmicb.2019.00107 (2019).

12. Aziz, R. K. et al. The RAST server: Rapid annotations using subsystems technology. BMC Genomics 9, 75. https://doi.org/10.1186/ 1471-2164-9-75 (2008)

13. Kanehisa, M. \& Goto, S. KEGG: Kyoto encyclopedia of genes and genomes. Nucleic Acids Res. 28, 27-30. https://doi.org/10.1093/ nar/28.1.27 (2000)

14. Alazzam, B., Bonnassie-Rouxin, S., Dufour, V. \& Ermel, G. MCLMAN, a new minimal medium for Campylobacter jejuni NCTC 11168. Res. Microbiol 162, 173-179. https://doi.org/10.1016/j.resmic.2010.09.024 (2011).

15. Velayudhan, J. \& Kelly, D. J. Analysis of gluconeogenic and anaplerotic enzymes in Campylobacter jejuni: an essential role for phosphoenolpyruvate carboxykinase. Microbiology 148, 685-694. https://doi.org/10.1099/00221287-148-3-685 (2002).

16. Kawanishi, T. et al. New detection systems of bacteria using highly selective media designed by SMART: Selective medium-design algorithm restricted by two constraints. PLoS ONE 6, e16512. https://doi.org/10.1371/journal.pone.0016512 (2011).

17. Salahudeen, A. K., Clark, E. C. \& Nath, K. A. Hydrogen peroxide-induced renal injury. A protective role for pyruvate in vitro and in vivo. J. Clin. Invest. 88, 1886-1893. https://doi.org/10.1172/jci115511 (1991).

18. Kim, J. C., Oh, E., Kim, J. \& Jeon, B. Regulation of oxidative stress resistance in Campylobacter jejuni, a microaerophilic foodborne pathogen. Front. Microbiol. 6, 751. https://doi.org/10.3389/fmicb.2015.00751 (2015).

19. Benoni, R. et al. Modulation of Escherichia coli serine acetyltransferase catalytic activity in the cysteine synthase complex. FEBS Lett. 591, 1212-1224. https://doi.org/10.1002/1873-3468.12630 (2017).

20. Vorwerk, H. et al. Utilization of host-derived cysteine-containing peptides overcomes the restricted sulphur metabolism of Campylobacter jejuni. Mol. Microbiol. 93, 1224-1245. https://doi.org/10.1111/mmi.12732 (2014).

21. Dickgiesser, N. \& Czylwik, D. Chemically defined media for auxotyping of Campylobacter jejuni. Zentralbl. Bakteriol. Mikrobiol. Hyg. A 260, 57-64. https://doi.org/10.1016/S0176-6724(85)80098-5 (1985).

22. Chandrashekhar, K., Kassem, I. I. \& Rajashekara, G. Campylobacter jejuni transducer like proteins: Chemotaxis and beyond. Gut Microbes 8, 323-334. https://doi.org/10.1080/19490976.2017.1279380 (2017).

23. Li, Z. et al. Methyl-accepting chemotaxis proteins 3 and 4 are responsible for Campylobacter jejuni chemotaxis and jejuna colonization in mice in response to sodium deoxycholate. J. Med. Microbiol. 63, 343-354. https://doi.org/10.1099/jmm.0.068023-0 (2014).

24. Vegge, C. S., Brøndsted, L., Li, Y.-P., Bang, D. D. \& Ingmer, H. Energy taxis drives Campylobacter jejuni toward the most favorable conditions for growth. Appl. Environ. Microbiol. 75, 5308-5314. https://doi.org/10.1128/aem.00287-09 (2009).

25. Seong, P. N. et al. Characterization of chicken by-products by mean of proximate and nutritional compositions. Korean. J. Food. Sci. Anim. Resour. 35, 179-188. https://doi.org/10.5851/kosfa.2015.35.2.179 (2015).

26. Visscher, C. et al. Influence of a specific amino acid pattern in the diet on the course of an experimental Campylobacter jejuni infection in broilers. Poult. Sci. 97, 4020-4030. https://doi.org/10.3382/ps/pey276 (2018).

27. Adedokun, S. A., Adeola, O., Parsons, C. M., Lilburn, M. S. \& Applegate, T. J. Factors affecting endogenous amino acid flow in chickens and the need for consistency in methodology. Poult. Sci. 90, 1737-1748. https://doi.org/10.3382/ps.2010-01245 (2011).

28. Hoffman, P. S., George, H. A., Krieg, N. R. \& Smibert, R. M. Studies of the microaerophilic nature of Campylobacter fetus subsp. jejuni. II. Role of exogenous superoxide anions and hydrogen peroxide. Can. J. Microbiol. 25, 8-16. https://doi.org/10.1139/m79002 (1979).

29. Karmali, M. A. et al. Evaluation of a blood-free, charcoal-based, selective medium for the isolation of Campylobacter organisms from feces. J. Clin. Microbiol. 23, 456-459. https://doi.org/10.1128/JCM.23.3.456-459.1986 (1986).

30. Mendz, G. L., Ball, G. E. \& Meek, D. J. Pyruvate metabolism in Campylobacter spp. Biochim. Biophys. Acta 1334, 291-302. https:// doi.org/10.1016/S0304-4165(96)00107-9 (1997).

31. Verhoeff-Bakkenes, L., Arends, A. P., Snoep, J. L., Zwietering, M. H. \& de Jonge, R. Pyruvate relieves the necessity of high induction levels of catalase and enables Campylobacter jejuni to grow under fully aerobic conditions. Lett. Appl. Microbiol. 46, 377-382. https://doi.org/10.1111/j.1472-765X.2008.02326.x (2008).

32. Reilly, S. S. \& Gilliland, S. E. Improved culturing techniques for Campylobacter. J. Food Sci. 68, 2752-2757. https://doi.org/10. 1111/j.1365-2621.2003.tb05800.x (2003).

33. Davis, L. \& DiRita, V. Growth and laboratory maintenance of Campylobacter jejuni. Curr. Protoc. Microbiol. 8(8A), $11-8 \mathrm{~A} 17$. https://doi.org/10.1002/9780471729259.mc08a01s10 (2008).

34. Secker, D., Tompkins, D. \& Alderson, G. Gas-permeable lifecell tissue culture flasks give improved growth of Helicobacter pylori in a liquid medium. J. Clin. Microbiol. 29, 1060-1061. https://doi.org/10.1128/JCM.29.5.1060-1061.1991 (1991).

35. Rollins, D. M., Coolbaugh, J. C., Walker, R. I. \& Weiss, E. Biphasic culture system for rapid Campylobacter cultivation. Appl. Environ. Microbiol. 45, 284-289. https://doi.org/10.1128/AEM.45.1.284-289.1983 (1983).

36. Shadowen, R. D. \& Sciortino, C. V. Improved growth of Campylobacter pylori in a biphasic system. J. Clin. Microbiol. 27, 1744-1747. https://doi.org/10.1128/JCM.27.8.1744-1747.1989 (1989).

37. Sellars, M. J., Hall, S. J. \& Kelly, D. J. Growth of Campylobacter jejuni supported by respiration of fumarate, nitrate, nitrite, trimethylamine-n-oxide, or dimethyl sulfoxide requires oxygen. J. Bacteriol. 184, 4187-4196. https://doi.org/10.1128/jb.184.15. 4187-4196.2002 (2002).

38. Kaakoush, N. O., Miller, W. G., De Reuse, H. \& Mendz, G. L. Oxygen requirement and tolerance of Campylobacter jejuni. Res. Microbiol. 158, 644-650. https://doi.org/10.1016/j.resmic.2007.07.009 (2007).

39. Ghaffar, N., Connerton, P. \& Connerton, I. Filamentation of Campylobacter in broth cultures. Front. Microbiol. 6, 657. https://doi. org/10.3389/fmicb.2015.00657 (2015).

40. Wright, J. et al. Metabolite and transcriptome analysis of Campylobacter jejuni in vitro growth reveals a stationary-phase physiological switch. Microbiology 155, 80-94. https://doi.org/10.1099/mic.0.021790-0 (2009).

41. Skirrow, M. B. Encyclopedia of Food Sciences and Nutrition. 2nd Edn. (ed. Benjamin Caballero) 779-786 (Academic Press, 2003).

42. King, Y. T. \& Chen, T. C. Chemical and physical characteristics of chicken livers following adrenocorticotropic hormone-induced stress. J. Food. Sci. 63, 589-591. https://doi.org/10.1111/j.1365-2621.1998.tb15791.x (1998).

43. Mabelebele, M., John, A., Ngambi, J., Norris, D. \& Ginindza, M. Comparison of gastrointestinal tracts and pH values of digestive organs of Ross 308 broiler and indigenous Venda chickens fed the same diet. Asian. J. Anim. Vet. Adv. 9, 71-76. https://doi.org/ 10.3923/ajava.2014.71.76 (2014).

44. Ciurescu, G., Vasilachi, A., Habeanu, M. \& Dragomir, C. Effects of dietary lentil seeds inclusion on performance, carcass characteristics and cecal pH of broiler chickens. Indian J. Anim. Sci. 87, 1130-1134 (2017).

45. Zaefarian, F., Abdollahi, M. R., Cowieson, A. \& Ravindran, V. Avian liver: The forgotten organ. Animals (Basel) 9, 63. https://doi. org/10.3390/ani9020063 (2019).

46. Bolzani, R., Ruggeri, F. \& Olivo, O. M. Average normal temperature of the chicken in the morning and after 1-2 days of fasting. Boll. Soc. Ital. Biol. Sper. 55, 1618-1622 (1979).

47. Aroori, S. V., Cogan, T. A. \& Humphrey, T. J. The effect of growth temperature on the pathogenicity of Campylobacter. Curr. Microbiol. 67, 333-340. https://doi.org/10.1007/s00284-013-0370-1 (2013).

48. Kim, S. S. et al. The effect of the repeated subcultures of Helicobacter pylori on adhesion, motility, cytotoxicity, and gastric inflammation. J. Korean. Med. Sci. 17, 302-306. https://doi.org/10.3346/jkms.2002.17.3.302 (2002). 
49. Overbeek, R. et al. The SEED and the Rapid Annotation of microbial genomes using Subsystems Technology (RAST). Nucleic Acids Res. 42, 206-214. https://doi.org/10.1093/nar/gkt1226 (2014).

50. Altschul, S. F. et al. Gapped BLAST and PSI-BLAST: A new generation of protein database search programs. Nucleic Acids Res. 25, 3389-3402. https://doi.org/10.1093/nar/25.17.3389 (1997).

51. Phung, C. et al. Campylobacter hepaticus, the cause of Spotty Liver Disease in chickens: Transmission and routes of infection. Front. Vet. Sci. 6, 505. https://doi.org/10.3389/fvets.2019.00505 (2019).

\section{Acknowledgements}

CP was supported by a joint Vietnam International Education Development and RMIT Scholarship.

\section{Author contributions}

C.P. designed, conducted the study and carried out data analysis. R.J.M. and T.T.H.V. conceived and supervised the work. T.B.W., J.A.Q. and P.C.S. facilitated and conducted the animal trial. C.P. wrote the first draft of the manuscript and T.T.H.V. and R.J.M. critically reviewed the manuscript. All authors commented on the manuscript and approved the final version of the manuscript.

\section{Competing interests}

The authors declare no competing interests.

\section{Additional information}

Correspondence and requests for materials should be addressed to R.J.M.

Reprints and permissions information is available at www.nature.com/reprints.

Publisher's note Springer Nature remains neutral with regard to jurisdictional claims in published maps and institutional affiliations.

(c) (i) Open Access This article is licensed under a Creative Commons Attribution 4.0 International

License, which permits use, sharing, adaptation, distribution and reproduction in any medium or format, as long as you give appropriate credit to the original author(s) and the source, provide a link to the Creative Commons licence, and indicate if changes were made. The images or other third party material in this article are included in the article's Creative Commons licence, unless indicated otherwise in a credit line to the material. If material is not included in the article's Creative Commons licence and your intended use is not permitted by statutory regulation or exceeds the permitted use, you will need to obtain permission directly from the copyright holder. To view a copy of this licence, visit http://creativecommons.org/licenses/by/4.0/.

(C) The Author(s) 2021 